\title{
The Form of Representations of the Canonical Commutation Relations for Bose Fields and Connection with Finitely Many Degrees of Freedom*
}

\author{
G. C. Hegerfeldt and O. Melsheimer \\ Institut für Theoretische Physik, Universität Marburg \\ Received January 10, 1969
}

\begin{abstract}
Given a representation of the canonical commutation relations (CCR) for Bose fields in a separable (or, under an additional assumption, nonseparable) Hilbert space $\mathfrak{G}$ it is shown that there exists a decreasing sequence of finite and quasi-invariant measures $\mu_{n}$ on the space $\mathscr{V}^{\prime}$ of all linear functionals on the test function space $\mathscr{V}, \mu_{1} \geqq \mu_{2} \geqq \ldots$, such that $\mathfrak{H}$ can be realized as the direct sum of the $L_{\mu_{n}}^{2}$, the space of all $\mu_{n}$-square-integrable functions on $\mathscr{V}^{\prime}$. In this realization $U(f)$ becomes multiplication by $e^{i(F, f)}, F \in \mathscr{V}^{\prime}$. The action of $V(g)$ is similar as in the case of cyclic $U(f)$ which has been treated by ARAKI and GELFand. But different $L_{\mu_{n}}^{2}$ can be mixed now. Simply transcribing the resulis in terms of direct integrals one obtains a form of the representations which turns out to be essentially the direct integral form of LEw. All results are independent of the dimensionality of $\mathscr{V}$ and hold in particular for $\operatorname{dim} \mathscr{V}<\infty$. Thus one has obtained a form of the CCR which is the same for a finite and an infinite number of degrees of freedom. From this form it is in no way obvious why there is such a great distinction between the finite and infinite case. In order to explore this question we derive von Neumanns theorem about the uniqueness of the Schrödinger operators in a constructive way from this dimensionally independent form and show explicitly at which point the same procedure fails for the infinite case.
\end{abstract}

\section{A. Introduction}

Several branches of quantum field theory are based on the canonical (equal time) commutation relations between the field $\Phi(x)$ and the conjugate field $\pi(x)$. Putting $\boldsymbol{x}=\left(0, x_{1}, x_{2}, x_{3}\right)$ one demands

$$
\left[\Phi(\boldsymbol{x}), \pi\left(\boldsymbol{x}^{\prime}\right)\right]=i \delta^{(3)}\left(\boldsymbol{x}-\boldsymbol{x}^{\prime}\right)
$$

with the other commutators vanishing.

In order to treat the CCR in a mathematically rigorous manner, one has to regard the fields as operator-valued distributions on a test function space. This can be achieved by replacing $\Phi(\boldsymbol{x})$ and $\pi(\boldsymbol{x})$ by $\Phi(f), \pi(g)$ where $f, g$ are elements of a subspace $\mathscr{V}$ of all real square-integrable

* Part of this paper is contained in Section IV of the Habilitationsschrift "Aspekte der kanonischen Vertauschungsrelationen für Quantenfelder" by G. C. HEGERFELDT, University of Marburg 1968. 
functions on $R^{3}$ and where $\Phi(f), \pi(g)$ can be regarded heuristically as smeared field operators,

$$
\Phi(f)=\int \Phi(\boldsymbol{x}) f(\boldsymbol{x}) d^{3} x, \quad \pi(g)=\int \pi(\boldsymbol{x}) g(\boldsymbol{x}) d^{3} x .
$$

In order to avoid domain questions connected with unbounded operators one introduces the unitary Weyl operators $U(f)$ and $V(g)$ whose formal relationship to the fields is given by

$$
U(f)=e^{i \Phi(f)}, \quad V(g)=e^{i \pi(g)} .
$$

Using Eqs. (1.2) and (1.3) one obtains by formal calculation from Eq. (1.1)

$$
\begin{aligned}
U\left(f_{1}\right) U\left(f_{2}\right) & =U\left(f_{1}+f_{2}\right), V\left(g_{1}\right) V\left(g_{2}\right)=V\left(g_{1}+g_{2}\right), \\
V(g) U(f) & =e^{i(f, g)} U(f) V(g)
\end{aligned}
$$

where $(f, g)$ is the usual scalar product in $\mathscr{V}$. Unitarity of $U(f)$ and $V(g)$ implies

$$
\begin{aligned}
U(0) & =V(0)=1, \\
U(f)^{*} & =U(-f), V(g)^{*}=V(-g) .
\end{aligned}
$$

Equations (1.4) and (1.5) are usually taken as a starting point for a rigorous investigation of the CCR. It should be pointed out, however, that there are more representations of the fields than of the Weyl operators since the transition from Eq. (1.2) to Eq. (1.4) may not be possible in a well-defined mathematical way. But, under a continuity assumption, one can always obtain the fields $\Phi(f), \pi(g)$ from $U(f), V(g)$ by STone's theorem. The continuity condition imposed in this paper is the customary one. For fixed $f$ and $g$ it is assumed that $U(\lambda f)$ and $V(\lambda g)$ are weakly continuous in $\lambda$. This implies strong continuity and the (weak and strong) continuity of $U\left(\sum_{1}^{n} \lambda_{i} f_{i}\right)$ and $V\left(\sum_{1}^{m} \lambda_{i} g_{i}\right)$ in $\lambda_{i}$ for fixed $f_{i}, g_{i}$.

We note that the CCR of quantum mechanics are contained in Eq. (1.4). To see this take $\mathscr{V}$ to be finite-dimensional, $\operatorname{dim} \mathscr{V}=n$ say, and introduce an orthonormal basis $h_{1}, \ldots, h_{n}$ in $\mathscr{V}$. For any $f, g \in \mathscr{V}$ one can write $f=\sum \alpha_{j} h_{j}, g=\beta_{j} h_{j}$. Defining $Q_{j}$ and $P_{j}$ by

$$
U\left(\lambda h_{j}\right)=e^{i \lambda Q_{j}}, V\left(\lambda h_{j}\right)=e^{i \lambda Q_{j}}
$$

via Stone's theorem, one obtains

$$
U(f)=e^{i \Sigma \alpha_{j} Q_{j}}, V(g)=e^{i \Sigma \beta_{j} P_{j}} .
$$

The $Q_{j}, P_{j}$ obey the usual commutation relations. Thus one can treat representations of the CCR for a finite number and an infinite number of degrees of freedom (fields) on equal footing.

The structure of representations of the CCR for fields has been investigated in several papers. We mention in particular the results of 
Araki [1], Gelfand [2], and Lew [3]. The first named author showed that representations with cyclic $U(f)$ can be realized by means of a finite quasi-invariant measure $\mu$ on the space $\mathscr{V}^{\prime}$ of all linear functionals on $\mathscr{V}$. In this realization the Hilbert space consists of the space $L_{\mu}^{2}$ of all $\mu$-square-integrable functions $\varphi(F)$ on $\mathscr{V}^{\prime} . U(f)$ is given by multiplication by $e^{i(F, f)}$ and $V(g)$ by multiplication with a certain function $a_{g}(F)$ and simultaneous translation of $\varphi(F)$ by $g^{1}$. In [2] the same construction has been carried through for nuclear spaces $\mathscr{V}$ and cyclic $U(f)$ which are continuous in the nuclear topology. In this case one can confine oneself to continuous functionals. Lew has shown in a very long paper that any representation of the CCR in a separable Hilbert space can be realized by means of a direct integral with a quasi-invariant measure on a space which is somewhat larger than $\mathscr{V}^{\prime}$. Under certain restrictions on $U(f)$ this result can be carried over to non-separable Hilbert spaces.

In this paper we first derive in a fairly short way an alternative realization of the general case in terms of a direct sum of Hilbert spaces. We show that there exists a decreasing sequence of finite and quasiinvariant measures $\mu_{n}$ on $\mathscr{V}^{\prime}, \mu_{1} \geqq \mu_{2} \geqq \ldots$, such that the Hilbert space $\mathfrak{G}$ can be realized as the direct sum of the $L_{\mu_{n}}^{2}$ and such that $U(f)$ is given by multiplication with $e^{i(F, f)}$. The action of $V(g)$ is similar to the cyclic case, but now different $L_{\mu_{n}}^{2}$ can be mixed by $V(g)$. The derivation is based on a generalization of BOCHNER's theorem for positive-definite continuous functions $[1,2]$ already used in the case of cyclic $U(f)$. The main task is to show that all measures can be chosen to be quasiinvariant and to determine the form of $V(g)$. This is carried through in Section B. At the end of Section B it is shown that by means of the measure $\mu_{1}$ one can write $\mathfrak{S}$ as a direct integral of Hilbert spaces $\mathfrak{S}(F)$. Simply transcribing our results we get a direct integral form of the representation. This turns out to be essentially the form of LEw, the only difference being that the measure is defined on the smaller space $\mathscr{V}^{\prime}$. Thus, as a by-product, one has obtained a fairly short derivation of LEW's result.

Our results are independent of the number of degrees of freedom. However, according to von NeumanN's theorem [4] every representation of the CCR for finitely many degrees of freedom is unitarily equivalent to a direct sum of Schrödinger representations. Thus, in spite of the same structural form of the representations, there exist uncountably many inequivalent irreducible ones in the infinite case while, as soon as $\mathscr{V}$ is finite-dimensional, all irreducible representations become equivalent.

${ }^{1}$ Every element $g \in \mathscr{V}$ defines a linear functional $F_{g}$ on $\mathscr{V}$ by means of the scalar product in $\mathscr{V},\left(F_{g}, f\right)=(g, f)$. Identifying $F_{g}$ and $g$ one can consider $\mathscr{V}$ as a subspace of $\mathscr{V}^{\prime}, \mathscr{V} \subset \mathscr{V}^{\prime}$. 
The reason for this strong degeneracy cannot be understood by the original proof of von NEUMANN because it admits of no parallels to the infinite case. Recently there appeared another proof [5] of von NEUManN's theorem which could have been of help for this question. Unfortunately it contains an error at a central place, and it is not obvious how to cure this. Another, earlier sketch of a proof [6] which runs on the same lines as [5] apparently contains this error also.

Therefore, in order to see explicitly why the case of finitely many degrees of freedom is so simple in comparison to the infinite case, we start from the dimensionally independent realization of the CCR by measures on $\mathscr{V}^{\prime}$, specialize this to finite-dimensional test function spaces, and step by step exploiting their particular properties we obtain in a constructive way von Neumann's result. The reduction is based on certain measure theoretic properties of the field operators. It turns out that the simplicity of the finite case is due to two facts which make a carrying over of our construction to the infinite case impossible. Firstly, for $\operatorname{dim} \mathscr{V}=n<\infty$ there exists, up to equivalence, only one quasi-invariant measure. And secondly, equally important, in the finite case the space $\mathscr{V}^{\prime}$ adjoint to $\mathscr{V}$ is equal to $\mathscr{V}$ while in the infinite case $\mathscr{V} \neq \mathscr{V}^{\prime}$ holds. This allows several inequivalent representations for one measure.

\section{B. Form of the Field Operators}

\section{Case of Cyclic $U(f)$}

First we briefly review representations of the CCR in which $U(f)$ is cyclic. This case has been treated in [1] and [2].

Let $\varphi$ be some fixed vector in $\mathfrak{G}$, and define a complex-valued function $E(f)$ on $\mathscr{V}$ by

$$
E(f)=\langle\varphi, U(f) \varphi\rangle .
$$

Then $E(f)$ is a positive functional on $\mathscr{V}$. Due to the assumed continuity of the representation $E\left(\sum_{1}^{m} \lambda_{i} f_{i}\right)$ is continuous in the real variables $\lambda_{1}, \ldots, \lambda_{m}$ for fixed $f_{0}, \ldots, f_{m} \in \mathscr{V}$. As a generalization of a theorem of Bochner [7], ARAKI [1] has shown that for any such continuous positive functional $E(f)$ on $\mathscr{V}$ there exists a (countably additive, nonnegative) finite measure $\mu$ on the Borel sets of the space $\mathscr{V}^{\prime}$ of all linear functionals ${ }^{2} F$ on $\mathscr{V}$ such that $E(f)$ is the Fourier transform of $\mu$,

$$
E(f)=\int_{\mathscr{V}^{\prime}} e^{i(F, f)} d \mu(F) .
$$

The Borel sets of $\mathscr{V}^{\prime}$ are generated by the cylinder sets of $\mathscr{V}^{\prime}$. For finitedimensional $\mathscr{V}$ one deals with the usual Borel sets in Euclidean spaces. of $\mathscr{V}$.

2 i.e., algebraically all, not only those which are continuous in some topology 
A more detailed exposition of these questions can be found in [2], [8], [9]. If $\mathscr{V}$ is a nuclear space and if $E(f)$ is continuous in the nuclear topology one can take $\mu$ to be a measure on the space of all continuous functionals [2]. For other topologies this need not be true.

Equation (2.2) is used for a realization of representations with cyclic $U(f)$. Let $\varphi_{0}$ be a vector cyclic for $U(f), f \in \mathscr{V}$, and take $\varphi=\varphi_{0}$ in Eqs. $(2.1-2)$. With each vector in $\mathfrak{G}$ of the form

one associates the function

$$
\psi=\sum_{K=1}^{m} \lambda_{K} U\left(f_{K}\right) \varphi_{0}
$$

$$
\varphi(F)=\sum_{1}^{m} \lambda_{K} e^{i\left(F, f_{K}\right)}
$$

in $L_{\mu}^{2}$. The mapping $\psi \rightarrow \psi\left(F^{\prime}\right)$ is isometric, by Eq. (2.2). It can be extended isometrically to all vectors of $\mathfrak{g}$. Moreover, since also the set of all functions of the form of Eq. (2.4) is dense ${ }^{3}$ in $L_{\mu}^{2}$, one has obtained an isometric mapping of $\mathfrak{G}$ onto $L_{\mu}^{2}$. Obviously $\varphi_{0}(F) \equiv 1$.

To find the form of $U(f)$ in $L_{\mu l}^{2}$ induced by this mapping, one applies $U(f)$ to Eq. (2.3) and finds that $U(f)$ acts on a dense set in $L_{\mu}^{2}$ as multiplication by $e^{i(F, f)}$. Then, by continuity, this holds for every element $\varphi(F)$ of $L_{\mu}^{2}$.

To find the form of $V(g)$, denote by $a_{g}$ the image of $\varphi_{0}$ under $V(g)$, and let $a_{g}(F)$ be a representative function in the corresponding equivalence class in $L_{\mu}^{2}$. Applying $V(g)$ to vectors of the form of Eq. (2.3) one obtains by Eq. (1.4) and footnote ${ }^{1}$

$$
(V(g) \varphi)(F)=a_{g}(F) \sum_{1}^{m} \lambda_{K} e^{i\left(F+g, f_{K}\right)}=a_{g}(F) \varphi(F+g) .
$$

One has to exercise some care when extending this relation to all elements of $L_{\mu}^{2}$ by denseness arguments. For any $\varphi \in L_{\mu}^{2}$ there exists a sequence of functions $\psi_{\alpha}(F)$ of the form of Eq. (2.4) converging in norm to $\varphi(F)$. One can further assume that the $\psi_{\alpha}(F)$ converge to $\varphi(F)$ pointwise almost everywhere (a. e.), i. e., $\psi_{\alpha}\left(F^{\prime}\right) \rightarrow \varphi\left(F^{\prime}\right)$ for $F \notin \mathfrak{R}$, where $\mathfrak{I}$ is a $\mu$-null set. By the norm convergence of $\psi_{\alpha}$ one knows that

$$
\left\|V(g) \psi_{\alpha}-V(g) \varphi\right\| \rightarrow 0 \text { for } \alpha \rightarrow \infty .
$$

On the other hand,

$$
\left(V(g) \psi_{\alpha}\right)(F)=a_{g}(F) \psi_{\alpha}(F+g) .
$$

3 Since the Borel sets in $\mathscr{V}^{\prime}$ are generated by the cylinder sets in $\mathscr{V}^{\prime}$ it suffices that characteristic functions of cylinder sets can be approximated. Going over to the finite-dimensional factor spaces $\mathscr{V}^{\prime} / W$ associated with these cylinder sets one is led to usual finite-dimensional Borel measures (cf., e.g., [9]) for which the completeness of the exponential functions follows from a theorem on Fourier transform of finite Borel measures [10]. 
Now, from the convergence in norm of $\psi_{\alpha}$ one cannot conclude that also the translated functions $\psi_{\alpha}^{(g)}(F)=\psi_{\alpha}(F+g)$ converge in norm. Therefore, in order to determine the form of the limit, we use the pointwise convergence of $\psi_{\alpha}(F)$ to $\varphi(F)$ for $F \notin \mathcal{R}$.

Now, in order to conclude that

$$
(V(g) \varphi)(F)=a_{g}(F) \varphi(F+g)
$$

a. e. it suffices that $2 \mathfrak{l}-g$ is a $\mu$-null set, i. e., that $\mu$ is $(\mathscr{V}-)$ quasiinvariant $^{4}$. To show this consider

$$
\left\langle\varphi_{0}, U(f) \varphi_{0}\right)=\int e^{i(F+g, f)} d \mu(F+g) .
$$

Due to the unitarity of $V(g)$ this equals

$$
\left\langle V(g) \varphi_{0}, V(g) U(f) \varphi_{0}\right\rangle=e^{i(g, f)} \int e^{i(F, f)}\left|a_{g}\left(F^{\prime}\right)\right|^{2} d \mu(F) .
$$

Hence $d \mu(F+g)$ and $\left|a_{g}(F)\right|^{2} d \mu(F)$ possess the same Fourier transform and give therefore ${ }^{5}$ rise to the same measure. Integrating over $\mathfrak{i}$ one obtains $\mu(\mathfrak{Z}+g)=0$.

From $V\left(g+g^{\prime}\right)=V(g) V\left(g^{\prime}\right)$ one obtains for almost all $F$

$$
a_{g+g^{\prime}}(F)=a_{g}(F) a_{g^{\prime}}(F+g) .
$$

For every finite quasi-invariant measure $\mu$ on the Borel sets of $\mathscr{V}^{\prime}$ there exists conversely at least one unitary representation of the CCR with cyclic $U(f)$. Choose $L_{\mu}^{2}$ as Hilbert space, define $U(f)$ as multiplication by $e^{i(F, f)}$ and $V(g)$ as in Eq. (2.6) with $a_{g}(F)=\{d \mu(F+g) / d \mu(F)\}^{1 / 2}$.

\section{General Case as a Direct Sum}

Now the theorem of ARAKI for positive functionals, Eq. (2.2), will be applied to the general case. First we note the following simple lemma (cf. [11]).

Lemma 2.1. Let $G$ be a group and $D(\mathfrak{g}), \mathfrak{g} \in G$, be a unitary representation of $G$ in a Hilbert space $\mathfrak{G}$. Then $\mathfrak{S}$ decomposes into a (possibly uncountable) direct sum of invariant subspaces in which the representation $D$ is cyclic. If $\mathfrak{H}$ is separable then the sum is countable.

A separable Hilbert space $\mathfrak{Y}$ can be written as a countable direct sum of subspaces $\mathfrak{G}_{n}$ in which $U(f)$ is cyclic. For each $n$ let $\varphi_{n}$ be a cyclic vector in $\mathfrak{G}_{n}$ with $\left\|\varphi_{n}\right\| \leqq 1$. Then $E_{n}(f)=\left\langle\varphi_{n}, U(f) \varphi_{n}\right\rangle$ is a positive functional on $\mathscr{V}$, and Eq. (2.2) applies to $E_{n}(f)$ with a measure $\mu_{n}$. As before each $\mathfrak{S}_{n}$ is mapped isometrically onto $L_{\mu_{n}}^{2}$, and under this mapping $U(f)$ becomes multiplication by $e^{i(F, f)}$ in $\mathfrak{G}=\sum_{n} \oplus L_{\mu_{n}}^{2}$.

4 One can also obtain the form of $V(g)$ directly, without the quasi-invariance (ARAKI, private communication).

5 This follows from the completeness of the exponential functions, or, as in footnote 3 , directly from the corresponding theorem for finite-dimensional spaces in $[10]$. 
Now put $\mu=\sum_{n} 2^{-n} \mu_{n}$. Obviously $\mu(\Delta)=0$ implies $\mu_{n}(\Delta)=0$ for each $n$. The Radon-Nikodym derivative $\eta_{n}(F)=d \mu_{n}(F) / d \mu(F)$ is uniquely determined up to a set of $\mu$-measure zero. Define $\mathfrak{A}_{n}=\left\{F \in \mathscr{V}^{\prime} ; \eta_{n}(F) \neq 0\right\}$. One has $\mu_{n}(\mathfrak{Q})=\left\|\varphi_{n}\right\|^{2} \leqq 1, \mu_{n}\left(\mathscr{V}^{\prime}-\mathfrak{A}_{n}\right)=0$. Let $\varphi\left(F^{\prime}\right)$ be a function in $L_{\mu_{n}}^{2}$. Then the mapping $\varphi(F) \rightarrow \varphi(F)\left\{\eta_{n}(F)\right\}^{1 / 2}$ is an isometric mapping from $L_{\mu_{n}}^{2}$ onto the space $L_{\mu}^{2}\left(\mathfrak{A}_{n}\right)$ of all functions in $L_{\mu}^{2}$ vanishing outside $\mathfrak{A}_{n}$. Thus one can write $\mathfrak{G}=\sum_{n} \oplus L_{\mu}^{2}\left(\mathfrak{A}_{n}\right)$, and $U(f)$ is still the multiplication by $e^{i(F, f)}$.

By induction one can show that one can assume the $\mathfrak{A}_{n}$ to be ordered by inclusion, $\mathfrak{A}_{1} \supset \mathfrak{A}_{2} \supset \ldots$, corresponding to a minimal number of measures. Defining measures $\tilde{\mu}_{n}$ on $\mathscr{V}^{\prime}$ by $\tilde{\mu}_{n}(\mathfrak{B})=\mu\left(\mathfrak{A}_{n} \cap \mathfrak{B}\right)$, one trivially has $L_{\tilde{\mu}_{n}}^{2}\left(\mathscr{V}^{\prime}\right)=L_{\mu}^{2}\left(\mathfrak{A}_{n}\right)$. Then $\mu=\tilde{\mu}_{1}$, and the other measures are restrictions of $\mu$ to $\mathfrak{A}_{n}$. One therefore can assume from the beginning that the decomposition of $\mathfrak{G}$ has these properties. We summarize this in the next lemma.

Lemma 2.2. If $\mathfrak{G}$ is separable, or if $\mathfrak{G}$ is a direct countable sum of subspaces which are cyclic for $U(f)$, then one can decompose $\mathfrak{G}$ as

$$
\mathfrak{H}=\sum_{n} \oplus L_{\mu_{n}}^{2}
$$

in such a way that $\mu=\mu_{1}$ is normed and such that the $\mu_{n}$ are restrictions of $\mu$ to a decreasing sequence of subsets $\mathfrak{A}_{n}$ of $\mathscr{V}^{\prime}, \mathscr{V}^{\prime}=\mathfrak{A}_{1} \supset \mathfrak{A}_{2} \supset \ldots, \mu_{n}(\mathfrak{B})$ $=\mu\left(\mathfrak{A}_{n} \cap \mathfrak{B}\right)=\int_{\mathfrak{B}} \chi_{n}(F) d \mu(F)$, where $\chi_{n}(F)$ is the characteristic function of $\mathfrak{A}_{n}$. In each $L_{\mu_{n}}^{2}$ the operator $U(f)$ is given by multiplication by $e^{i(F, f)}$.

To determine the form of $V(g)$ in this decomposition, let $\varphi_{n}^{0} \in \mathfrak{S}$ be the vector with the constant function 1 as component in $L_{\mu_{n}}^{2}$ and zero components otherwise. Denote by $a_{g}{ }_{n}^{m}$ the component of $V(g) \varphi_{n}^{0}$ in $L_{\mu_{m}}^{2}$. Let $\psi^{(n)}$ be a vector of the form

$$
\begin{aligned}
\psi^{(n)} & =\sum_{1}^{M} \lambda_{K} U\left(f_{K}\right) \varphi_{n}^{0}, \\
\left(\psi^{(n)}\right)^{m}(F) & =\delta_{n m} \psi^{n}(F)=\delta_{n m} \sum_{1}^{M} \lambda_{K} e^{i\left(F, j_{K}\right)} .
\end{aligned}
$$

Analogously to Eq. (2.5) one finds

$$
\left(V(g) \psi^{(n)}\right)^{m}(F)=a_{g n}^{m}(F) \psi^{n}(F+g) .
$$

We extend this relation to all elements of $L_{\mu_{n}}^{2}$. A sequence of functions converging in $L_{\mu_{n}}^{2}$ need not converge in $L_{\mu_{m}}^{2}$ for $m<n$, and in Eq. (2.12) the product on the r.h.s., which belongs to $L_{\mu_{m}}^{2}$, contains a function from $L_{\mu_{n}}^{2}$. So we first consider characteristic functions $\chi_{\Delta}\left(F^{\prime}\right)$ where $\Delta$ is a Borel set in $\mathscr{V}^{\prime}$. Since $\chi_{\Delta} \in L_{\mu_{n}}^{2}$ for every $n$, in particular $\chi_{\Delta} \in L_{\mu}^{2}$, there exists a sequence of functions $\psi_{\alpha}(F)$ of the form (2.11) converging in $L_{\mu}^{2}$ to $\chi_{A}$. 
This implies convergence in each $L_{\mu_{n}}^{2}$. One can assume that $\psi_{\alpha}(F)$ converges to $\chi_{\Delta}(F)$ pointwise $\mu$-almost everywhere, i.e., for $F \notin \mathfrak{R}$ with $\mu(\mathfrak{V})=0$. Let $\chi_{\Delta}^{(n)}, \psi_{\alpha}^{(n)} \in \mathfrak{S}$ be vectors with $n$-th component $\chi_{\Delta}$ and $\psi_{\alpha}$, respectively, and zero otherwise. Then $\left(V(g) \chi_{\Delta}^{(n)}\right)^{m}(F)$, the component of $V(g) \chi_{A}^{(n)}$ in $L_{\mu_{n}}^{2}$, is the limit in the norm of $L_{\mu_{m}}^{2}$ of $\left(V(g) \psi_{\alpha}^{(n)}\right)^{m}=a_{g n}^{m}(F)$ $\times \psi_{\alpha}(F+g)$ for $\alpha \rightarrow \infty$. To find the form of this limit we proceed as before. For $F \notin \mathfrak{T}-g$ one has by pointwise convergence

$$
\left(V(g) \psi_{\Delta}^{(n)}\right)^{m}(F)=a_{g}^{m}(F) \chi_{\Delta}(F+g) .
$$

To obtain an equality of both sides in $L_{\mu_{m}}^{2}$, one needs that $\mathfrak{i}-g$ is a $\mu_{m}$-null set. For this it suffices that $\mu(\mathfrak{R}-g)=0$, i.e., that $\mu$ is quasiinvariant. This will be shown further below. With this proviso one has obtained the form of $V(g)$ for characteristic functions, and the transition to arbitrary elements of $L_{\mu_{n}}^{2}$ is immediate.

It remains to show the quasi-invariance of $\mu$. The unitarity of $V(g)$ implies

$$
\left\langle\varphi_{m}^{0}, U(f) \varphi_{n}^{0}\right\rangle=e^{i(f, g)}\left\langle V(g) \varphi_{m}^{0}, U(f) V(g)(g) \varphi_{n}^{0}\right\rangle .
$$

Similar to Eqs. (2.7) and (2.8) this leads to

$$
\delta_{n m} \int e^{i\left(F^{\prime}, f\right)} d \mu_{n}(F+g)=\sum_{v} \int e^{i\left(F^{\prime}, f\right)} \bar{a}_{g}{ }_{m}(F) a_{g n}^{\nu}(F) d \mu_{v}(F) .
$$

By Lemma 2.2 one has $d \mu_{v}(F)=\chi_{v}(F) \cdot d \mu(F)$. Inserting this and noting that now one can interchange sum and integral ${ }^{6}$ one obtains an equality for Fourier transforms of (complex-valued) measures. Therefore ${ }^{5}$

$$
d \mu_{n}(F+g)=\sum_{v}\left|a_{g}{ }_{n}(F)\right|^{2} \chi_{\nu}(F) d \mu(F)
$$

and for $m \neq n$

$$
\sum_{v} \bar{a}_{g}{ }_{m}^{\nu}(F) a_{g}^{\nu}(F) \chi_{\nu}(F)=0
$$

a.e., i.e., for $F \notin \mathfrak{T}=\mathfrak{T}(g)$ with $\mu(\mathfrak{T})=0$. $\mathfrak{i}$ can be taken to be independent of $n, m$. For a $\mu$-null set $\Delta$ Eq. (2.16) when integrated over $\Delta$ leads to $\mu_{n}(\Delta+g)=0$ for each $n$. Hence $\mu=\mu_{1}$ is quasi-invariant?. One can also show the following important result.

Lemma 2.3. All measures $\mu_{n}$ of Lemma 2.2 are quasi-invariant.

${ }^{6}$ If the sum is infinite, Eq. (2.15), with $m=n$ and $f=0$ implies the convergence of $\sum_{\nu}\left|a_{g_{n}}^{v}(F)\right|^{2} \chi_{\nu}(F)$ for $\mu$-almost all $F$. From this it follows that $\sum_{\nu} \bar{a}_{g m}^{\nu}(F) \cdot a_{g}{ }^{\nu}(F) \chi_{v}(F)$ converges for $\mu$-almost all $F$. Since

$$
\left|e^{i(F, f)} \cdot \sum_{\nu} \bar{a}_{g m}^{\nu}(F) a_{g n}^{\nu}\left(F^{\prime}\right) \chi_{\nu}(F)\right| \leqq\left(\sum_{\nu}\left|a_{g m}^{\nu}(F)\right|^{2} \chi_{\nu}\right)^{1 / 2} \cdot\left(\sum_{\nu}\left|a_{g m}^{\nu}\left(F^{\prime}\right)\right|^{2} \chi_{\nu}\right)^{1 / 2},
$$

one can apply Lebesgue's bounded convergence theorem.

7 To determine the form of $V(g)$ one actually does not need that the measures $\mu_{n}$ are ordered as in Lemma 2.2. Up to now only the quasi-invariance of $\mu$ has been used, and this follows from Eq. (2.16) if one writes $d \mu_{n}(F+g)=\chi_{n}(F+g)$ $\times d \mu(F+g)$ and if one decomposes $\Delta$ into disjoint parts lying in $\mathfrak{A}_{n}-g, n=1,2 \ldots$ 
Proof. We use induction on $n$. For $n=1, \mu_{1}=\mu$ is quasi-invariant. Assume $\mu_{m}$ to be quasi-invariant for $m=1, \ldots, n-1$. Let $\mu_{n}(\Delta)=0$. It suffices to consider Borel sets $\Delta$ which lie in $\mathfrak{A}_{n}^{R} \equiv \mathfrak{A}_{n-1}-\mathfrak{A}_{n}$, since on $\mathfrak{A}_{n} \mu_{n}$ coincides with $\mu$ and since outside of $\mathfrak{A}_{n-1}$ also $\mu_{n-1}$ vanishes, the quasi-invariance of which has been assumed as induction hypothesis. So let $\Delta \subset \mathfrak{A}_{n}^{R}$. For any $F \in \mathfrak{A}_{n}^{R}$ one has $\chi_{v}(F)=0$ for $v \geqq n$ and $\chi_{v}(F)=1$ otherwise. Therefore, for any $\Omega \subset \mathfrak{A}_{n}^{R}$ and arbitrary $m$, one obtains by Eq. (2.16)

$$
\mu_{m}(\Omega+g)=\int_{\Omega} \sum_{\nu=1}^{n-1}\left|a_{g}^{\nu}(F)\right|^{2} d \mu(F) .
$$

Equation (2.17) becomes for $F \in \mathfrak{A}_{n}^{R}, F^{\prime} \notin \mathfrak{R}(g)$ and any $m, m^{\prime}$ with $m \neq m^{\prime}$

$$
\sum_{\nu=1}^{n-1} \bar{a}_{g m}^{\nu}(F) a_{g}^{\nu} m_{m^{\prime}}(F)=0
$$

As a first step we are going to show that there exists a $\mu$-null set $\widetilde{\mathfrak{I}}=\widetilde{\mathfrak{I}}(g)$ such that for $m=1,2, \ldots, n-1$

$$
\sum_{\nu=1}^{n-1}\left|a_{g}^{v}(F)\right|^{2} \neq 0 \quad \text { for } \quad F \in \mathfrak{A}_{n}^{R}, F \notin \widetilde{\mathfrak{Q}} .
$$

Indeed, let $\mathfrak{B}_{m}=\left\{F \in \mathfrak{A}_{n}^{R}, \sum_{\nu=1}^{n-1}\left|a_{g m}^{\nu}(F)\right|^{2}=0\right\}$. Then, by Eq. (2.18), $\mu_{m}\left(\mathfrak{B}_{m}+g\right)=0$. The quasi-invariance of $\mu_{m}$ for $m<n$ implies $\mu_{m}\left(\mathfrak{B}_{m}\right)=0$. Since on $\mathfrak{Q}_{n}^{R} \mu_{m}$ and $\mu$ coincide for $m<n$, one has $\mu\left(\mathfrak{B}_{m}\right)=0$. Putting $\widetilde{\mathfrak{I}}=\bigcup_{m} \mathfrak{B}_{m}$ one obtains Eq. (2.20).

Now consider, for fixed $F \in \mathfrak{Q}_{n}^{R}$ and for $m<n$, the $n-1$ row vectors

$$
\left(a_{g m}^{1}(F), \ldots, a_{g} \underset{m}{n-1}(F)\right) \text {. }
$$

Equations (2.19) and (2.20) state that for $F \notin \mathfrak{l}(g) \cup \widetilde{\mathfrak{I}}$ these $n-1$ vectors are mutually orthogonal and nonzero, hence they are linearly independent. Putting $m^{\prime}=n$ in Eq. (2.19) one sees that for $F \notin \mathfrak{R}(g)$ the row vector $\left(a_{g n}^{1}(F), \ldots, a_{g n}^{n-1}(F)\right)$ is orthogonal to these. It follows that for $v=1, \ldots, n-1$

$$
a_{g}^{\nu}(F)=0 \quad \text { for } \quad F \in \mathfrak{Q}_{n}^{R}, F^{\prime} \notin \mathfrak{R}(g) \cup \widetilde{\mathfrak{Z}},
$$

i.e., $\mu$-almost everywhere on $\mathfrak{A}_{n}^{R}$. Hence, putting $m=n$ and $\Omega=\Delta$ in Eq. (2.18), one obtains $\mu_{n}(\Delta+g)=0$. q.e.d.

The quasi-invariance of $\mu_{n}$ and $\mu_{n}\left(\mathscr{V}^{\prime}-\mathfrak{A}_{n}\right)=0$ imply by Eq. (2.16) that $a_{g}{ }_{n}^{\nu}(F) \cdot \chi_{v}(F)=0 \mu$-almost everywhere on $\mathscr{V}^{\prime}-\mathfrak{A}_{n}$. Hence one can insert the factor $\chi_{n}(F)$ on the r.h.s. of Eq. (2.16). With $d \mu_{n}=\chi_{n} d \mu$ one obtains

$$
d \mu_{n}(F+g)=\sum_{\nu}\left|a_{g}^{\nu}\left(F^{\prime}\right)\right|^{2} \chi_{v}\left(F^{\prime}\right) d \mu_{n}\left(F^{\prime}\right)
$$

and thus an expression for the Radon-Nikodym derivative

$$
d \mu_{n}(F+g) / d \mu_{n}(F) \text {. }
$$


When generalizing Eq. (2.12) to all vectors of $\mathfrak{S}$ one has to distinguish between different kinds of convergence. Any $\varphi \in \mathfrak{G}$ can be written as a finite or infinite sum of vectors $\varphi^{(n)}$ which possess only in $L_{\mu_{n}}^{2}$ a nonvanishing component, $\varphi^{n}(F)$ say. Therefore

$$
(V(g) \varphi)^{m}(F)=\text { l.i.m. } \sum_{n} a_{g}^{m}(F) \varphi^{n}(F+g) \text { in } L_{\mu_{m}}^{2},
$$

where the limit in the mean on the r.h.s. is a limit in the norm of $L_{\mu_{m}}^{2}$. We now prove that the sum on the r.h.s. converges even pointwise $\mu_{m}$-almost everywhere.

Lemma 2.4. Let $\varphi \in \mathfrak{G}$, with component $\varphi^{n}$ in $L_{\mu_{n}}^{2}$. Then for $\mu_{m^{-}}$ almost all $F$

or, for $\mu$-almost all $F$,

$$
(V(g) \varphi)^{m}(F)=\sum_{n} a_{g}^{m}(F) \varphi^{n}(F+g),
$$

$$
(V(g) \varphi)^{m}(F)=\sum_{n} a_{g n}^{m}(F) \chi_{m}(F) \varphi^{n}(F+g),
$$

where the sum, if infinite, is to be understood as a pointwise limit.

Proof. In case of a finite sum the statement is trivial. For an infinite sum it follows as in footnote ${ }^{6}$ that $\sum_{n}\left|\varphi^{n}(F)\right|^{2} \chi_{n}(F)$ converges for $F$ not in some $\mu$-null set $\mathfrak{P}_{\varphi}$. Denote by $\chi_{n}^{\prime}(F)$ the characteristic function of $\mathscr{V}^{\prime}-\mathfrak{A}_{n}$. Then $\chi_{n}^{\prime}\left(F^{\prime}\right)$ vanishes $\mu_{n}$-almost everywhere, and Eq. (2.13) implies that $a_{g}^{m}\left(F^{\prime}\right) \cdot \chi_{n}^{\prime}(F+g)$ vanishes $\mu_{m}$ - a.e., hence

$$
a_{g n}^{m}(F) \chi_{m}(F) \chi_{n}^{\prime}(F+g)=0
$$

$\mu$-a.e.,i.e., for $F$ not in some $\mu$-null set $\mathfrak{R}_{g, 1}$ which may be assumed to be independent of $n, m$. Hence for $F \notin \mathfrak{Z}_{g, 1}$

$$
a_{g}^{m}(F) \chi_{m}(F) \varphi^{n}(F+g)=a_{g}^{m}(F) \chi_{m}(F) \chi_{n}(F+g) \varphi^{n}(F+g),
$$

and to prove Eq. (2.24) it suffices to show that $\sum_{\nu}\left|a_{g}{ }_{\nu}^{m}(F)\right|^{2} \chi_{m}(F)$ converges $\mu$ - a.e. To this end let $\chi_{a}^{(m)} \in \mathfrak{S}$ be a vector with the characteristic function $\chi_{\Delta}(F)$ as $m$-th component and zero otherwise. Unitarity implies

$$
\left\langle\chi_{\Delta}^{(m)}, V(g) \varphi_{\nu}^{0}\right\rangle=\left\langle V(-g) \chi_{\Delta}^{(m)}, \varphi_{\nu}^{0}\right\rangle .
$$

Evaluating this in terms of integrals the arbitrariness of $\Delta$ yields

$a_{g v}^{m}(F) \chi_{m}(F)=\bar{a}_{-g m}^{v}(F+g) \chi_{v}(F+g) \frac{d \mu(F+g)}{d \mu(F)} \quad \mu$-a.e.,

for $F$ not in some null set $\mathfrak{R}_{g, 2}$. By Eqs. (2.16) and (2.17), with $\mathfrak{R}(g)$ the respective null set, one obtains

$\sum_{\nu} \bar{a}_{g \nu}^{m}(F) a_{g \nu}^{n}\left(F^{\prime}\right) \chi_{m}\left(F^{\prime}\right) \chi_{n}\left(F^{\prime}\right)=\delta_{n m} \chi_{n}\left(F^{\prime}\right) \frac{d \mu(F+g)}{d \mu(F)} \quad \mu$-a.e.,

for $F \notin \mathfrak{R}_{g, 3} \equiv \mathfrak{R}_{g, 2} \cup(\mathfrak{Z}(-g)-g)$. Thus one has in particular pointwise convergence for $m=n$ and $F \notin \mathfrak{I}_{g, 3}$ which is again a null set. Hence the sum in Eq. (2.24) converges pointwise for $F \notin \mathfrak{Z}_{g, 3} \cup\left(\mathfrak{Z}_{p}-g\right)$. $\quad$ q.e.d. 
The equality $V(g) V\left(g^{\prime}\right) \varphi_{n}^{0}=V\left(g+g^{\prime}\right) \varphi_{n}^{0}$, considered in each $L_{l_{m}}^{2}$. yields as an analogue to Eq. (2.9)

$$
\text { l.i.m. } \sum_{v} a_{g}^{m}(F) a_{g^{\prime} n}^{\nu}(F+g)=a_{g+g^{\prime}}{ }_{n}^{m}(F) \text { in } L_{\mu_{m}}^{2} .
$$

Noting that $a_{g n}^{\nu}(F+g)$ is a particular $\phi^{\nu}(F+g)$ the first part of Lemma 2.4 shows that the sum converges pointwise for $F$ not in some $\mu_{m}$-null set $\mathfrak{2}_{g, g^{\prime}}\left(\mu_{m}\right)$,

$$
a_{g+g^{\prime} n}^{m}(F)=\sum_{\nu} a_{g_{v}}^{m}(F) a_{g^{\prime} n}^{\nu}(F+g) \quad \mu_{m} \text { - a.e. }
$$

The following theorem summarizes our results.

Theorem 1. Let $U(f), V(g)$ be a unitary representation of the CCR in a separable ${ }^{8}$ Hilbert space $\mathfrak{G}$, with $f, g \in \mathscr{V}$. Then there exist quasi-invariant measures $\mu_{1} \geqq \mu_{2} \geqq \ldots$ on the Borel sets of $\mathscr{V}^{\prime}$, where $\mu_{1}$ is normed and each $\mu_{n}$ is a restriction of the preceding measures to a subset $\mathfrak{A}_{n}$ of $\mathscr{V}^{\prime}$, such that $\mathfrak{S}$ can be realized as

$$
\mathfrak{S}=\sum_{n} \oplus L_{\mu_{n}}^{2}
$$

with the action of $U(f)$ and $V(g)$ on a vector $\varphi$ with components $\varphi^{n}$ in $L_{\mu_{n}}^{2}$ given by

$$
\begin{aligned}
& (U(f) \varphi)^{m}(F)=e^{i(F, f)} \varphi^{m}(F) \\
& (V(g) \varphi)^{m}(F)=\sum_{n} a_{g n}^{m}(F) \varphi^{n}(F+g) \quad \mu_{m} \text { - a.e., }
\end{aligned}
$$

with pointwise convergence $\mu_{m}$-almost everywhere. The functions $a_{g}{ }_{n}^{m}(F)$ satisfy Eq. (2.28), and $d \mu_{n}(F+g) / d \mu_{n}(F)$ is given by Eq. (2.22). Conversely, for any set of such quasi-invariant measures there exists a unitary representation of the CCR.

Sometimes one allows the test function spaces for $U(f)$ and $V(g)$ to be different, $f \in \mathscr{V}_{f}$ and $g \in \mathscr{V}_{g}$ say. The above construction is easily carried over to this more general case. The only change is that $\mathscr{V}^{\prime}$ is replaced by $\mathscr{V}_{f}^{\prime}$ and that the measures are $\mathscr{V}_{g}$-quasi-invariant, i. e., under translation by $g \in \mathscr{V}_{g}$. It is also obvious that the above construction is immediately carried over to multi-component fields.

\section{General Case as a Direct Integral}

Theorem 1 can easily be rewritten in direct integral form. This will lead essentially to the form of representations of the CCR obtained by LEW [3].

Consider the $1_{2}$-space of sequences of complex numbers,

$$
\left(z^{1}, z^{2}, \ldots\right), \sum_{v}\left|z^{v}\right|^{2}<\infty .
$$

${ }^{8}$ From the derivation it is clear that the same theorem holds also for a nonseparable Hilbert space $\mathfrak{Y}$ if $\mathfrak{G}$ decomposes into a countable direct sum of subspaces which are cyclic for $U(f)$. 
With each $F \in \mathscr{V}^{\prime}$ associate a subspace $\mathfrak{S}(F)$ of $1_{2}$ consisting of those sequences which in addition satisfy

$$
z^{n}=0 \quad \text { if } \quad F \notin \mathfrak{A}_{n} .
$$

From the quasi-invariance of $\mu_{n}$ it follows that $\mu_{n}\left(\mathfrak{A}_{n}-g\right)=\mu_{n}\left(\mathfrak{A}_{n}\right)$ and hence $\mu_{n}\left(\mathfrak{A}_{n} \cap\left(\mathfrak{U}_{n}-g\right)\right)=\mu_{n}\left(\mathfrak{A}_{n}\right)$. Therefore $\mathfrak{A}_{n}-g$ and $\mathfrak{A}_{n}$ differ at most by a $\mu$-null set, and consequently

This implies

$$
\chi_{\mathscr{A}_{n}-g}(F) \equiv \chi_{n}(F+g)=\chi_{n}(F) \quad \mu \text { - a.e. }
$$

$$
\operatorname{dim} \mathfrak{S}(F)=\operatorname{dim} \mathfrak{S}(F+g) \quad \mu \text {-a.e., }
$$

i.e., for $F$ not in some $\mu$-null set $2_{d}(g)$.

For each $\psi \in \mathfrak{G}$ with components $\psi^{n}$ in $L_{\mu_{n}}^{2}$ define a function $\psi(F)$ on $\mathscr{V}^{\prime}$ with values in $\mathfrak{G}(F)$ for $\mu$-almost all $F$ by (cf. [12])

$$
\Psi(F)=\left(\psi^{1}(F) \chi_{1}(F), \psi^{2}(F) \chi_{2}(F), \ldots\right) .
$$

That condition (2.29) is satisfied for $\mu$-almost all $F$ follows from footnote 6 . Denote for each $F$ the norm of $\Psi(F)$ by $\|\Psi(F)\|$, and define

$$
\|\Psi\|^{2}=\int\|\Psi(F)\|^{2} d \mu(F) .
$$

Then obviously $\|\Psi\|=\|\psi\|$, and one has obtained a realization of $\mathfrak{G}$ as a direct integral. In this realization $U(f)$ acts as multiplication by $e^{i(F, f)}$ and $V(g)$ as

where

$$
(V(g) \Psi)(F)=\left(\sum_{n} a_{g}^{\prime}{ }_{n}^{1}(F) \Psi^{n}(F+g), \ldots\right) \quad \text { a.e. }
$$

$$
a_{g n}^{\prime m}(F)=a_{g n}^{m}(F) \chi_{m}(F)
$$

and where $\Psi^{n}(F)$ is the $n$-th component of $\Psi(F)$. The sums in Eq. (2.32) converge $\mu$ - a.e.

Consider the matrix function

$$
B_{g}(F)=\left(a_{g k}^{\prime} i(F)\right) \cdot\left\{\frac{d \mu(F+g)}{d \mu(F)}\right\}^{1 / 2} .
$$

From Eq. (2.33) one has $a_{g k}^{\prime} i(F)=0$ for $i>n(F)$, and from Eqs. (2.25) and (2.30) $a_{g}^{\prime} i(F)=0$ for $k>n(F)$ and $F$ not in the exceptional null sets of those equations. Hence, for $F$ not in some null set $\mathfrak{R}_{g, 4}, B_{g}(F)$ is nontrivial only for this $n(F) \times n(F)$ submatrix. Eqs. $(2.16-2.17)$ and (2.27), together with Eq. (2.30), show that this submatrix is unitary for $F$ not in some null set. Since $\mathfrak{S}(F+g)$ and $\mathfrak{S}(F)$ are equal for $F \notin \mathfrak{R}_{d}(g)$, by Eq. (2.31), $B_{g}(F)$ can be considered as a unitary operator from $\mathfrak{G}(F+g)$ onto $\mathfrak{G}(F)$ for $\mu$-almost all $F$. Eq. (2.28) becomes

$$
B_{g}(F) B_{g^{\prime}}(F+g)=B_{g+g^{\prime}}(F) \quad \text { a.e., }
$$

i.e., for $F$ not in some null set $\mathfrak{P}_{g, g^{\prime}}$. The results for the direct integral formulation can be summarized as follows. 
Theorem 2. Let $U(f), V(g)$ be a unitary representation of the $C C R$ in a separable ${ }^{8}$ Hilbert space $\mathfrak{S}$ with $f, g \in \mathscr{V}$. Then there exists a quasiinvariant normed measure $\mu$ on the Borel sets of $\mathscr{V}^{\prime}$ and a direct integral decomposition of $\mathfrak{G}$,

$$
\mathfrak{H}=\int_{\oplus} \mathfrak{H}(F) d \mu(F)
$$

with the following properties.

1. For given $g \in \mathscr{V}$ one has for $\mu$-almost all $F \in \mathscr{V}^{\prime}$

$$
\operatorname{dim} \mathfrak{S}(F)=\operatorname{dim} \mathfrak{H}(F+g) .
$$

2. Let $\psi \in \mathfrak{S}$, and let $\Psi(F)$ be the corresponding vector function. Then $U(f), V(g)$ are given by

$$
\begin{aligned}
& (U(f) \Psi)(F)=e^{i\left(F^{\prime}, f\right) \Psi(F)} \\
& (V(g) \Psi)(F)=\left\{\frac{d \mu(F+g)}{d \mu(F)}\right\}^{1 / 2} B_{g}(F) \Psi(F+g),
\end{aligned}
$$

where $B_{g}$ is a measurable operator function on $\mathscr{V}^{\prime}$ and where, for $\mu$-almost all $F, B_{g}(F)$ is a unitary operator from $\mathfrak{H}(F+g)$ onto $\mathfrak{H}(F)$. For any $g, g^{\prime} \in \mathscr{V}$ Eq. (2.34) holds. Each $\mathfrak{S}(F)$ can be realized as $1_{2}$-space of all sequences $\left(z^{1}, z^{2}, \ldots z^{n}, 0, \ldots\right)$ with $n=n(F)$ being the dimension of $\mathfrak{S}(F)$.

This is essentially the form of LEw [3] (representations satisfying the condition of footnote 8 are called countable there). The only difference is that instead of $\mathscr{V}^{\prime}$ the somewhat larger space $\mathscr{V}_{\infty}^{\prime}$ is used where $\mathscr{V}_{\infty}^{\prime}$ consists of all (real) linear functionals on $\mathscr{V}$ taking on finite or infinite values. To see the connection with a finite number of degrees of freedom $\mathscr{V}^{\prime}$ is more suitable. Again the results can be carried over to different test function spaces for $U(f)$ and $V(g)$.

\section{Connection with Finitely Many Degrees of Freedom}

\section{Specialization to Finite-Dimensional $\mathscr{V}$}

Theorems 1 and 2 hold both for finite- and infinite-dimensional $\mathscr{V}$. In the former case one knows from von NEUMANN's theorem that all representations decompose into a direct sum of Schrödinger representations. That this is so is in no way obvious from the general form, and it is also not obvious why there is such a great distinction between the CCR with finite- and infinite-dimensional $\mathscr{V}$. In the following we are therefore going to explore this question. By deriving voN NEUMANN's theorem from the results of Section 2 we intend to show the peculiarities of the finite case which prevent the carrying over of the derivation to the infinite case. We start from Theorem 1, but Theorem 2 can be used as well.

For $\operatorname{dim} \mathscr{V}=N<\infty \mathscr{V}$ is a finite-dimensional real Euclidean space, and therefore $\mathscr{V}^{\prime}=\mathscr{V}=R^{N}$. Introducing an orthonormal basis $h_{1}, \ldots, h_{\mathcal{N}}$ and denoting the coordinates of a general element by $x=\left(x^{1}, \ldots, x^{N}\right)$ 
one can consider the elements $f, g \in \mathscr{V}$ to be coordinate row vectors, similarly for the linear functionals. Therefore we replace $F$ by $x$ in the preceding formulas. The differential of the Lebesgue measure induced by the scalar product in $R^{N}$ will be denoted by $d^{N} x, d^{N} x=d x^{1} \ldots d x^{N}$.

The first important restriction now comes from the quasi-invariance of the measures $\mu_{n}$. For a finite-dimensional space any such measure is equivalent to the Lebesgue measure [13], which means that there exist functions $\varrho_{n}(x)$ positive almost everywhere and satisfying

$$
d \mu_{n}(x)=\varrho_{n}(x) d^{N} x .
$$

Since the measures $\mu_{n}$ are restrictions of $\mu$ it follows from the positivity of $\varrho_{n}(x)$ that all $\mu_{n}$ are equal,

$$
d \mu_{n}(x)=d \mu(x)=\varrho(x) d^{N} x .
$$

As in the general case one can now go over to the Lebesgue measure since only the equivalence class of the measures matters. But for one such class there could still be many inequivalent representations, due to a possible freedom in the choice of the $a_{g}{ }_{n}(x)$. The main task therefore consists in showing that for finite-dimensional $\mathscr{V}$ any possible choice of the $a_{g}^{n}(x)$ leads to a direct sum of Schrödinger representations and why in the infinite case an analogous result does not hold. In order to retain the connection to the infinite case we use $d \mu(x)$ instead of $\varrho(x) d^{N} x$ as long as possible.

It is clear that one has to exploit the functional Eq. (2.28). Define $b_{g} m(x)$ by

$$
a_{g}^{m}(x)=\left\{\frac{d \mu(x+g)}{d \mu(x)}\right\}^{1 / 2} \cdot b_{g}^{m}(x) .
$$

Then the matrix $B_{g}(x)=\left(b_{g} \frac{i}{k}(x)\right)$ is, by Eqs. (2.16), (2.17) and (2.27) unitary for almost all $x$, i.e., for $x$ not in some null set $\mathfrak{R}=\mathfrak{R}(g)$. Eq. (2.28) now reads

$$
B_{g}(x) B_{g^{\prime}}(x+g)=B_{g+g^{\prime}}(x) \quad \text { a.e. }
$$

i.e., for $x$ not in some null set $2\left(g, g^{\prime}\right)$.

KrRILLOv [6] has briefly sketched how one can obtain von NEUMaNN's theorem starting from a direct integral realization of the Hilbert space of quantum mechanics (cf. also [5]). He is led to the same functional relation as in Eq. (3.4), and putting $x=0$ finds

$$
B_{g^{\prime}}(g)=B_{g}(0)^{*} B_{g+g^{\prime}}(0) .
$$

From this he concludes in two lines von NEumanN's result. However, Eq. (3.5) is in general not true since Eq. (3.4) is an almost-everywhere equality with the exceptional set depending on $g, g^{\prime}$. The underlying misunderstanding in [6] as well as in [5] seems to be due to considering $L_{\mu}^{2}$ as a space of functions and not as a space of equivalence classes of 22 Commun.math.Phys., Vol.12 
functions. In the equivalence classes belonging to $V(g) \varphi_{n}^{0}$ one could have chosen representatives $a_{g}^{m}(x)$ which equal any prescribed numerical value for $x=0$, thus one could choose $b_{g}^{m}(0)=\delta_{n m}$ for all $g$. Then Eq. (3.5), if correct, would mean that $B_{g}(x)$ is always the unit matrix. Similarly one could make $B_{g}(x)$ vanish indentically. Furthermore, since $2 i\left(g, g^{\prime}\right)$ may depend on $g, g^{\prime}$ there may exist no $x_{0}$ such that Eq. (3.4) holds for all or almost all $g, g^{\prime}$. If one has such an $x_{0}$ one would get an expression similar to Eq. (3.5). That this cannot hold in general follows immediately from the fact that Eq. (3.5) implies that $B_{g}(x)$ is measurable in $g$ for fixed $x$ which is certainly not the case for an arbitrary selection of the representatives $B_{g}(x)$. The same remarks apply to the existence of the (unitary) inverse of $B_{g}(x)$.

However, further below it will be shown that one only has to know that the $B_{g}(x)$ can be chosen to possess a structural form similar to Eq. (3.5), and in this sense Eq. (3.5) gives an important heuristic hint. The following lemma will allow an immediate proof of von NEUMANN's result.

Lemma 3.1. There exist a matrix $\beta(x)$ consisting of measurable functions $\beta_{n}^{m}(x)$ and unitary a.e., a null set $\mathfrak{R}_{\beta}^{(2)}$ in $R^{N} \times R^{N}$, and a particular choice of the $B_{g}(x)$ such that

$$
B_{g}(x)=\beta^{*}(x) \beta(x+g) \quad \text { for } \quad(x, g) \notin \mathfrak{R}(2) .
$$

Nothing more than this form of $B_{g}(x)$ is needed. The proof of Eq. (3.6) is based on the next three lemmas pertaining to measure theoretic properties of the $b_{g}^{m}(x)$.

\section{Measure Theoretic Properties of the Operators $B_{g}(x)$}

Now it will be shown that the $a_{g n}^{m}(x)$ and $B_{g}(x)$ can be chosen to fulfil additional properties ${ }^{9}$.

Lemma 3.2. There exist measurable functions $b_{n}^{m}(x, g)$ on $R^{N} \times R^{N}$ and a null set $\mathfrak{R}_{r}$ such that for any choice of the $b_{g}{ }_{n}^{m}(x)$ one has

$$
b_{n}^{m}(x, g)=b_{g}{ }_{n}^{m}(x) \quad \text { for } \quad g \notin \mathfrak{R}_{r}, x \notin \mathfrak{I}^{b}(g)
$$

where ${ }^{2}{ }^{b}(g)$ is a null set depending on $g$ (and on the choice of $b_{g}^{m}(x)$ ).

Proof. Let $E^{\prime}, E^{\prime \prime}$ be measurable sets in $R^{N}$, and let $\chi_{E^{\prime}}, \chi_{E^{\prime \prime}}$ be the corresponding characteristic functions. Denote by $\chi_{E^{\prime}}^{(m)}$ the vector in $\oplus L_{\mu_{n}}^{2}$ whose components are $\chi_{E^{\prime}}$ in $L_{\mu_{m}}^{2}$ and zero otherwise. Consider

$$
\left\langle\chi_{E^{\prime}}^{(m)}, V(g) \varphi_{n}^{0}\right\rangle=\int_{R^{N}} \chi_{E^{\prime}}(x) a_{g}^{m}(x) d \mu(x) .
$$

${ }^{9}$ ARAKI has pointed out to us that in the context of induced representations such measurability questions have been investigated by Mackey (for Lemma 3.2 cf. in particular Duke Math. J. 16, 313 (1949)). 
The 1.h.s. is a bounded continuous function of $g$. Hence the integral over $d \mu(g)$ exists, in particular

$$
\nu\left(E^{\prime} \times E^{\prime \prime}\right) \equiv \int d \mu(g) \chi_{E^{\prime \prime}}(g) \int d \mu(x) \chi_{E^{\prime}}(x) a_{g}^{m}(x)
$$

is well-defined. Denote by $\mathfrak{W}$ the algebra of sets in $R^{N} \times R^{N}$ obtained by finite unions and differences of product sets in $R^{N} \times R^{N}$. It is clear that $v$ can be extended to a finitely additive set function on $\mathfrak{M}$. Let $E_{0}$ and $F_{1} \subset F_{2} \ldots$ be in $\mathfrak{M}$, and let $F_{n} \uparrow E_{0}$. By means of Schwarz's inequality and unitarity of $V(g)$ one obtains

$$
\left|\nu\left(E_{0}\right)-\nu\left(F_{n}\right)\right|^{2} \leqq \int d \mu(g) \int d \mu(x) \chi_{E_{0}-F_{n}}(x, g) .
$$

For $F_{n} \uparrow E_{0}$ this tends to 0 . Hence $\nu$ is countably additive on $\mathfrak{W}$.

Therefore $v$ can be uniquely extended by the Hahn extension [14] to a countably additive set function on the $\sigma$-algebra determined by $\mathfrak{R}$, i.e., on the Borel sets of $R^{N} \times R^{N}$. If $E$ is a $\mu \times \mu$-null set in $R^{N} \times R^{N}$, Eq. (3.10) implies $v(E)=0$.

Hence there exists a measurable function $a_{n}^{m}(x, g)$ on $R^{N} \times R^{N}$, the Radon-Nikodym derivative of $v$ with respect to $\mu \times \mu$ [15] such that for each Borel set $E$ in $R^{N} \times R^{N}$

$$
v(E)=\int_{E} d(\mu \times \mu)(x, g) a_{n}^{m}(x, g) .
$$

Applying FubINI's theorem to the r.h.s. of this relation one obtains for product sets $E^{\prime} \times E^{\prime \prime}$

$$
\int_{E^{\prime \prime}} d \mu(g)\left\{\int_{E^{\prime}} d \mu(x) a_{g n}^{m}(x)\right\}=\int_{E^{\prime \prime}} d \mu(g)\left\{\int_{E^{\prime}} d \mu(x) a_{n}^{m}(x, g)\right\} .
$$

Since this holds for all $E^{\prime \prime} \subset R^{N}$, the terms in curly brackets are equal for almost all $g$, where the exceptional null set $\mathfrak{2}_{E^{\prime}}$ may depend on $E^{\prime}$. In order to get rid of this dependence let first $E^{\prime}$ be a rational rectangle in $R^{N}$, i.e., an $N$-rectangle with sides parallel to the coordinate axes and with rational coordinates for the end points. Denote by $\mathfrak{Z}_{r}$ the union of all such $\mathfrak{V}_{E^{\prime}}$. Then $\mathfrak{T}_{r}$ is again a null set. Since each $N$-rectangle is a union of countably many rational $N$-rectangles, the equality holds for all measurable sets if $g \notin \mathcal{I}_{r}$. This implies that for $g \notin \mathfrak{I}_{r}$ the integrands are a.e. equal, where the exceptional null set $\mathfrak{R}(g)$ can depend on $g$. Putting $b_{n}^{m}(x, g)=\{d \mu(x+g) / d \mu(x)\}^{-1 / 2} \cdot a_{n}^{m}(x, g)$ this becomes Eq. (3.7). The null sets may be assumed to be independent of $m, n$. q.e.d.

The previous lemma means that for $g \notin \mathfrak{N}_{r}$ we can take $a_{n}^{m}(x, g)$ and $b_{n}^{m}(x, g)$ as representatives for $a_{g}^{m}(x)$ and $b_{g}{ }_{n}^{m}(x)$, respectively. Hence the matrix $B(x, g)=\left(b_{k}^{i}(x, g)\right)$ satisfies Eq. (3.4) for $g, g^{\prime}, g+g^{\prime} \notin \mathfrak{R}_{r}$ and $x$ not in some null set $\mathfrak{i}\left(g, g^{\prime}\right)$. The matrix is furthermore unitary for $g \notin \mathfrak{R}_{r}$ and $x \notin \mathfrak{R}(g)$. We now show that these properties can be sharpened in the following way. 
Lemma 3.3. There exists a null set $\mathfrak{Q}_{u}^{(2)}$ in $R^{N} \times R^{N}$ such that for $(x, g) \notin \mathfrak{R}_{u}^{(2)}$ the matrix $\left(b_{k}^{i}(x, g)\right.$ is unitary.

Proof. For each $g \notin \mathfrak{Z}_{r}$, there exists an $\mathfrak{R}(g)$ such that for $x \notin \mathfrak{R}(g)$

$$
\begin{aligned}
& \sum_{\alpha} \bar{b}_{m}^{\alpha}(x, g) b_{n}^{\alpha}(x, g)=\delta_{n m}, \\
& \sum_{\alpha} \bar{b}_{\alpha}^{m}(x, g) b_{\alpha}^{n}(x, g)=\delta_{n m} .
\end{aligned}
$$

In order to proceed similarly as in the proof of Lemma 3.2 we have to show that the sums define measurable functions. This is only a question of convergence for arbitrary $x$ and $g$. For $m=n$ all terms are positive so that in this case the sums define extended real-valued non-negative measurable functions. Integrating with respect to $x$ over $E^{\prime}$ and then with respect to $g$ over $E^{\prime \prime}$ and applying FuBINI's theorem one abtains in the same way as above that for $(x, g)$ not in some null set $22_{n}^{(2)} \subset R^{N} \times R^{N}$ the sums converge and equal 1. This implies that the sums in Eq. (3.13) converge for $(x, g) \notin \bigcup_{n} \mathfrak{i}_{n}^{(2)}$. Therefore, if $\tilde{\chi}(x, g)$ is the characteristic function of the complement of $\bigcup_{n} \mathfrak{q}_{n}^{(2)}$, one has

$$
\sum_{\alpha} \bar{b}_{m}^{\alpha}(x, g) b_{n}^{\alpha}(x, g) \tilde{\chi}(x, g)=\delta_{n m}
$$

for $(x, g) \notin \bigcup_{n} \mathfrak{Z}_{n}^{(2)}, g \notin \mathfrak{R}_{r}, x \notin \mathfrak{Z}(g)$, and the sum converges for all $(x, g)$, thus defining a measurable function on $R^{N} \times R^{N}$. Integrating with respect to $d \mu(x)$ and then with respect to $d \mu(g)$ over $E_{1}$ and $E_{2}$ one proceeds as before. q.e.d.

In a similar fashion Eq. (3.4) can be sharpened for $b_{k}^{i}(x, g)$ in the following way.

Lemma 3.4. There exists a null set $\mathfrak{2}^{(3)} \subset R^{N} \times R^{N} \times R^{N}$ such that for all $\left(x, g, g^{\prime}\right) \notin \mathfrak{I} i^{(3)}$ the matrices $\left(b_{k}^{i}(x, g)\right),\left(b_{k}^{i}\left(x+g, g^{\prime}\right)\right)$ and $\left(b_{k}^{i}\left(x, g+g^{\prime}\right)\right)$ are unitary and satisfy for all $m, n$

$$
\sum_{\alpha} b_{\alpha}^{n}(x, g) b_{m}^{\alpha}\left(x+g, g^{\prime}\right)=b_{m}^{n}\left(x, g+g^{\prime}\right)
$$

(convergence of the sum for $\left(x, g, g^{\prime}\right) \notin 2 \tau^{(3)}$ is implied).

Now we are in a position to prove Lemma 3.1.

Proof of Lemma 3.1. For almost all $x$, i.e., for $x$ not in some null set $\hat{\mathfrak{Z}}^{(1)}$,

$$
\mathfrak{R}_{x}^{(2)} \equiv\left\{\left(g, g^{\prime}\right) ;\left(x, g, g^{\prime}\right) \in \mathfrak{R}^{(3)}\right\}
$$

is a null set. Here $\mathfrak{2}^{(3)}$ is the null set of Lemma 2.4. In the same way one has for $x$ not in some null set $\widetilde{श}^{(1)}$ that

$$
\mathfrak{T}_{x}^{(1)} \equiv\left\{g ;(x, g) \in \mathfrak{Q}_{u}^{(2)}\right\}
$$

is a null set, where $\mathfrak{R}_{u}^{(2)}$ is the null set of Lemma 2.3. Let now $x_{0}$ be some fixed element not in $\hat{\mathfrak{I}}^{(1)} \cup \widetilde{\mathfrak{q}}^{(1)}$. Then the matrix $B\left(x_{0}, \tilde{g}\right)=\left(b_{k}^{i}\left(x_{0}, \tilde{g}\right)\right)$ 
is unitary for almost all $\tilde{g} \in R^{N}$, by Lemma 3.3, and by Lemma 3.4 one has for $\left(g, g^{\prime}\right) \notin \mathfrak{R}_{x_{0}}^{(2)} \cup\left(\mathfrak{R}_{x_{0}}^{(1)} \times R^{N}\right)$ that $B\left(x_{0}, g\right), B\left(x_{0}+g, g^{\prime}\right)$, and $B\left(x_{0}, g+g^{\prime}\right)$ are unitary and satisfy

$$
B\left(x_{0}, g\right) B\left(x_{0}+g, g^{\prime}\right)=B\left(x_{0}, g+g^{\prime}\right) .
$$

Hence for $\left(g, g^{\prime}\right) \notin \mathfrak{T}_{x_{0}}^{(2)} \cup\left(\mathfrak{V}_{x_{0}}^{(1)} \times R^{N}\right)$

$$
B\left(x_{0}+g, g^{\prime}\right)=B\left(x_{0}, g\right)^{*} B\left(x_{0}, g+g^{\prime}\right) .
$$

Put $\beta(x)=B\left(x_{0}, x-x_{0}\right)$ and

$$
\mathfrak{Q}^{(2)}=\left\{\left(x, g^{\prime}\right) ;\left(x-x_{0}, g^{\prime}\right) \in \mathscr{P}_{x_{0}}^{(2)} \cup\left(\mathfrak{T}_{x_{0}}^{(1)} \times R^{N}\right)\right\} .
$$

Then $\beta(x)$ is a unitary matrix for almost all $x$, and Eq. (3.14) can be rewritten as

$$
B\left(x, g^{\prime}\right)=\beta(x)^{*} \beta\left(x+g^{\prime}\right) \quad \text { for } \quad\left(x, g^{\prime}\right) \notin \mathfrak{Q}^{(2)} .
$$

Now, for $g \notin \mathfrak{Z}_{r}$ one can take $B(x, g)$ as a representative for $B_{g}(x)$, by Lemma 3.2. Hence, putting $\mathfrak{2}_{\beta}^{(2)}=\mathfrak{T}^{(2)} \cup\left(R^{N} \times \mathfrak{T}_{r}\right)$ one arrives at Lemma 3.1.

\section{Von Neumann's Theorem and Comparison with the Infinite Case}

In a similar way as in [6] where von NEuMaNN's theorem was deduced from the (unjustified) Eq. (3.5) one obtains this theorem from Lemma 3.1. If in the realization of $\mathfrak{S}$ as a direct sum, $\mathfrak{S}=\bigoplus_{n} L_{\mu_{n}}^{2}\left(R^{N}\right)$, the component of a vector $\psi \in \mathfrak{G}$ in $L_{\mu_{n}}^{2}$ is $\psi^{n}(x)$, one can associate $\psi$ with the vector function $\psi(x)=\left(\psi^{1}(x), \psi^{2}(x), \ldots\right)$, and one knows that for almost all $x$

Now put

$$
\|\psi(x)\|^{2}=\sum_{n}\left|\psi^{n}(x)\right|^{2}<\infty
$$

$$
\hat{\psi}(x)=\varrho(x)^{1 / 2} \beta(x) \psi(x)
$$

where $\varrho(x)$ is given by Eq. (3.2). Due to the unitarity of $\beta(x)$ for almost all $x$ one has

$$
\int\|\hat{\psi}(x)\| d^{N} x=\int \varrho(x)\|\beta(x) \psi(x)\|^{2} d^{N} x=\int\|\psi(x)\|^{2} d \mu(x) .
$$

Therefore the mapping $\psi(x) \leftrightarrow \hat{\psi}(x)$ defines an isometric mapping,

$$
\oplus L_{\mu_{n}}^{2}\left(R^{N}\right) \leftrightarrow \oplus L^{2}\left(R^{N}\right)
$$

It is clear that under this mapping $U(f)$ remains multiplication by $e^{i(x, f)}$.

To see what $V(g)$ becomes under this mapping, we note that for almost all $g$, i.e., $g$ not in some null set $\mathfrak{Z}^{\prime}$,

$$
\mathfrak{T}_{g}^{(1)} \equiv\left\{x:(x, g) \in \mathfrak{T}_{\beta}^{(2)}\right\}
$$


is a null set. So let $g \notin \mathfrak{R}^{\prime}$. Then Lemma 3.1 gives, for $x \notin \mathfrak{R}_{g}^{(1)}$ and $x$ not in the null set where $\beta(x)$ is possibly non-unitary,

$$
\begin{aligned}
(\hat{V}(g) \hat{\psi})(x) & =\varrho(x)^{1 / 2} \beta(x)(V(g) \psi)(x) \\
& =\varrho(x+g)^{1 / 2} \beta(x) \beta^{*}(x) \beta(x+g) \psi(x+g)=\hat{\psi}(x+g) .
\end{aligned}
$$

Thus $\hat{U}(f)$ and $\hat{V}(g)$, for $g \notin \mathcal{I}^{\prime}$, do not mix the different terms in the direct sum $\oplus L^{2}\left(R^{N}\right)$ and act in each as in the Schrödinger representation. Since $R^{N}-2 \mathfrak{2}^{\prime}$ is dense in $R^{N}$ it follows from the continuity of the representation that all $\hat{V}(g)$ have the Schrödinger form in each $L^{2}\left(R^{N}\right)$.

Up to now we have assumed that $\mathfrak{G}$ is separable. But since by Lemma 2.1 every representation is a (possibly uncountable) direct sum of cyclic representations and since for a cyclic representation of the CCR for finitely many degrees of freedom the Hilbert space is separable the problem is reduced to this case. Thus one has obtained vön NEuManN's theorem, that every continuous representation of the CCR for finitely many degrees of freedom in a separable or nonseparable Hilbert space is unitarily equivalent to a direct sum of Schrödinger representations.

If the representation is not continuous but only measurable one can show for a separable Hilbert space that the representation is also continuous [16]. Thus continuous can be replaced by measurable if $\mathfrak{S}$ is separable.

If the representation is cyclic with respect to $U(f)$ there is only one term in the decomposition of $\mathfrak{S}$ so that the representation is unitarily equivalent to the Schrödinger representation and therefore irreducible.

Now it is easy to see why the results cannot be taken over to the infinite case. First of all it has already been remarked that for $\operatorname{dim} \mathscr{V}=\infty$ there can exist many inequivalent quasi-invariant measures on $\mathscr{V}^{\prime}$. Representations belonging to inequivalent measures are, however, already inequivalent with respect to $U(f)$. Secondly, for one measure $\mu$ there can exist many possible $a_{g}(F)$ (or $a_{g}^{m}(F)$, respectively) leading to different inequivalent representations. All this is due to the fact that Lemma 3.1 for the form of $B_{g}(F)$ no longer holds. A part of the proof of this lemma could be carried over to the infinite case if one has an appropriate measure on $\mathscr{V}$. But the crucial point is Eq. (3.14). In order to get from $B\left(F_{0}+g, g^{\prime}\right)$ the form of $B\left(F, g^{\prime}\right)$ for all $F \in \mathscr{V}^{\prime}$ one needs that $g$ runs through the same set as $F$ does, i.e., through all of $\mathscr{V}^{\prime}$. But for $\operatorname{dim} \mathscr{V}=\infty$ one has $\mathscr{V} \varsubsetneqq \mathscr{V}^{\prime}$.

Summarizing the reasons for the great distinction between the CCR for a finite and an infinite number of degrees of freedom one can say that the richness for the infinite case is due to the existence of many inequivalent quasi-invariant measures and to the nonequality of $\mathscr{V}$ and $\mathscr{V}^{\prime}$. 
Acknowledgements. The autors would like to thank Professor G. LuDwig for his generous encouragement and Professor H. ARAKI and Dr. K. KraUs for critical remarks on the manuscript. One of us (G.C.H.) gratefully acknowledges financial support of the Bundesministerium für wissenschaftliche Forschung, Bonn.

\title{
References
}

1. Araki, H.: Hamiltonian formalism and the canonical commutation relations in quantum field theory. J. Math. Phys. 1, 492 (1960).

2. cf. Gelfand, I. M., and N. Y. Vilenkin: Generalized functions. Vol. 4, (last section). New York: Academic Press 1964.

3. LEw, I. S.: The structure of representations of the canonical commutation relations in quantum field theory. Thesis, Princeton University 1960 (unpublished).

4. Neumann, J. von: Die Eindeutigkeit der Schrödingerschen Operatoren. Math. Ann. 32, 191 (1931).

5. Straumann, N.: A new proof of von Neumann's theorem concerning the uniqueness of the Schrödinger operators. Helv. Phys. Acta 40, 518 (1967).

6. Kirmulov, A. A.: Unitary representations of nilpotent Lie groups. Russ. Math. Surveys 17, 53 (1962).

7. Bochner, S.: Vorlesungen über Fouriersche Integrale, (cf. p. 20). Leipzig: Akademische Verlagsgesellschaft 1932.

8. Kolmogorov, A.: Grundbegriffe der Wahrscheinlichkeitsrechnung, Ergebnisse der Mathematik, Bd. 2, No. 3. Berlin: Springer 1933.

9. Hegerfeldt, G. C.: Aspekte der kanonischen Vertauschungsrelationen für Quantenfelder, Habilitationsschrift, Universität Marburg 1968. Kernel integral formulas for the canonical commutation relations of quantum fields. I. Representations with cyclic field. To appear in J. Math. Phys. (1969).

10. Bochner, S.: Harmonic analysis and the theory of probability, (see Theorem 2.1.4, p. 24). Berkeley and Los Angeles: University of California Press 1960.

11. Neumark, M. A.: Normierte Algebren, (cf. p. 253). Berlin: VEB Deutscher Verlag der Wissenschaften 1959.

12. Natmark, M. A., and S. V. Fomin: Continuous direct sums of Hilbert spaces and some of their applications. Ann. Math. Soc. Transl. Ser. 2, 5, 35 (1957).

13. cf. [2], pp. $351-352$.

14. Dunford, N., and J. T. Schwarz: Linear operators, Part I, (p. 136). New York: Interscience 1958.

15. cf. [14], p. 181.

16. Riesz, F., and BéLA Sz.-NAgy: Vorlesungen über Funktionalanalysis (p. 370). Berlin: VEB Deutscher Verlag der Wissenschaften 1956.

\author{
( ${ }^{\prime}$. C. Hegerfeld \\ O. Melsheimer \\ Institut f. theoret. Physik \\ der Universität \\ 355 Marburg/Lahn \\ Renthof 7
}

